\title{
Psychometric properties of the Spanish version of the Sensitivity to Punishment and Sensitivity to Reward Questionnaire for Children (SPSRQ-C)
}

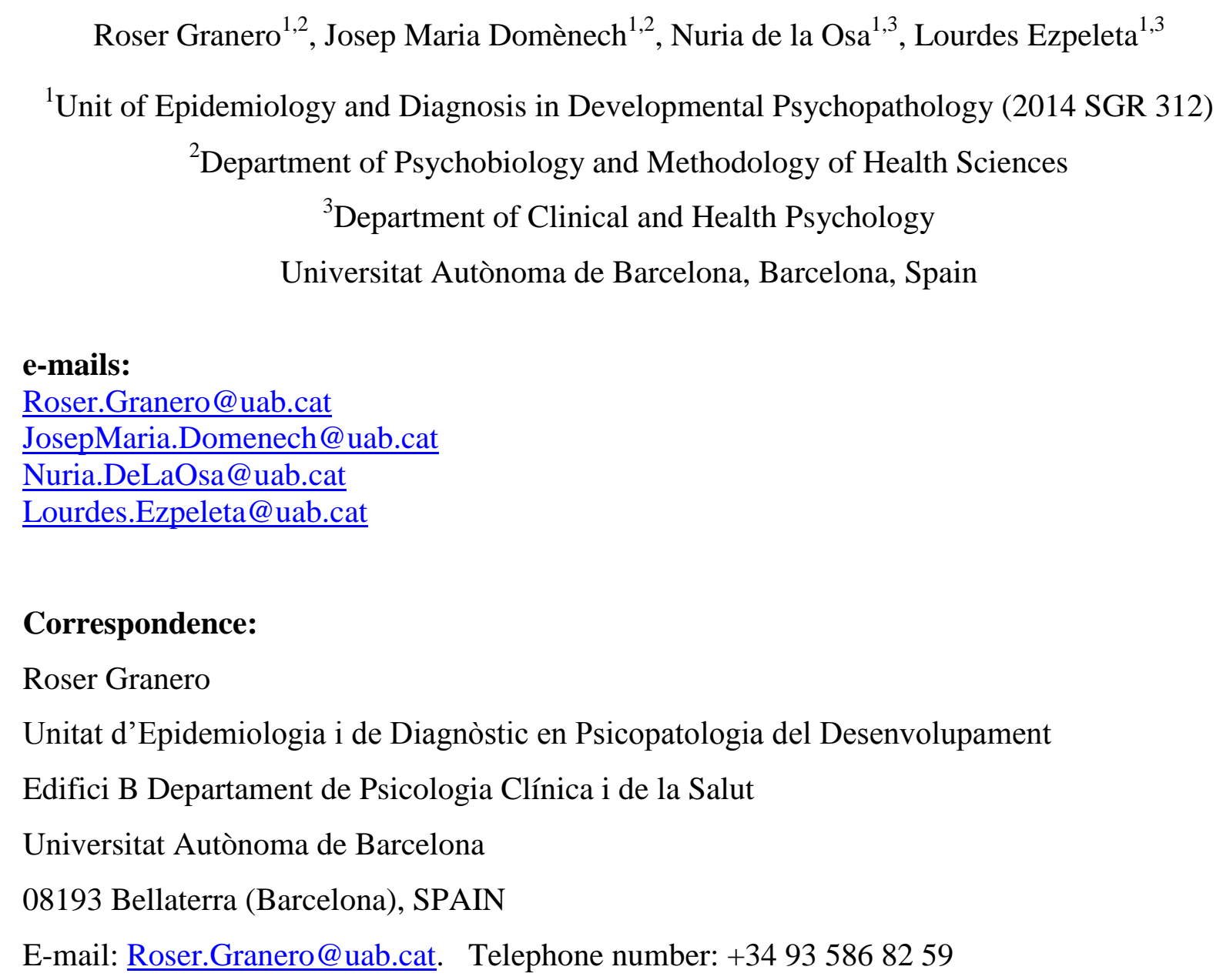

\section{Contributors:}

All authors have made substantial contributions to the work reported in the manuscript:

- Lourdes Ezpeleta designed the study and was responsible for assessment instruments.

- Roser Granero designed and conducted the statistical analysis, and wrote the first draft of the manuscript.

- All the authors have reviewed, contributed to and approved the final manuscript.

1 Conflict of interests. The authors have no conflicts of interests. 
Psychometric properties of the SPSRQ-C

\section{Highlights}

- Factorial analysis showed that the three-factor structure was optimal for the SPSRQ-

$\mathrm{C}$ in a large sample of 6-year-old children from the Spanish general population $(n=483)$.

- Convergent and discriminant validity was achieved between the three factors of the SPSRQ-C and the children's temperament traits (measured by questionnaire) and DSM-5 disorders (measured by diagnostic interview).

- The Spanish version of the SPSRQ-C has adequate psychometric properties as a measure of the sensitivity to punishment and reward responsivity based on RST in young children.

- Reliable and valid measurement of RST systems is relevant in the areas of educational and therapeutic interventions that use punishment and rewards as contingencies. Parent/teacher training programs may thus benefit especially. 


\section{1. Introduction}

\section{1.1. Formulation of Reinforcement Sensitivity Theory (RST)}

Jeffrey A. Gray proposed RST as a reformulation of the Eysenck model of human

4 personality (Gray, 1970, 1981). RST has recently been revised to incorporate findings from

5 different areas in psychology and neuroscience, and it constitutes at present a biologically based personality model that proposes the following three behavioral/neuropsychological systems to explain individual differences in the emotion, learning and motivation domains (Berkman, Lieberman \& Gable, 2009; Corr, 2004): a) a behavioral approach system (BAS, “appetitive system"), identified as an impulsivity trait, responsible for activating behaviors in the presence of reward and non-punishment signals; b) a behavioral inhibition system (BIS, “aversive system"), identified as an anxiety trait, responsible for inhibiting behavior in response to punishment, non-reward and novelty signals; and c) a flight-fight system (FFS, the "threat system"), responsible for mediating the subject's responses to aversive stimuli, novel stimuli and non-rewards.

\subsection{Measurement instruments based on RST for adults}

RST has been applied to different areas of adult psychological functioning, and at present a number of questionnaires exist to measure its three main systems (FFS, BIS and BAS). Many RST questionnaires in adulthood are based on the original BIS-BAS model, and were initially assessed through classical measures of anxiety and impulsivity (Corr, 2016). A specific first assessment measure for RST was the Gray-Wilson Personality Questionnaire (GWPG; Wilson, Barret \& Gray, 1989), which measured six components of rodent-reactions to reinforcement and covered the BAS, BIS and FFS system. This was followed by the General Reward and Punishment Expectancy Scales (GRAPES) (Ball \& Zuckerman, 1990), 
1 stimuli. Subsequently, Carver and White (1994) developed the BIS/BAS scales, divided into

2 three factors (impulsivity/fun-seeking, reward responsivity and drive). But the most consistent

3 attempt to develop a specific measurement tool within RST was the global self-report

4 proposed for adults by Torrubia, Avila, Moltó and Caseras (2001): the Sensitivity to

5 Punishment and Sensitivity to Reward Questionnaire (SPSRQ), a self-report with 48 yes-no

6 response items. In the SPSRQ, the Sensitivity to Punishment scale (24-items) was developed

7 to measure processes related to threat of punishment or failure (BIS), while the Sensitivity to

8 Reward scale (24-items) assessed differences in Gray's impulsivity dimension (BAS), with

9 FFS not being considered. But this original two-factor structure did not consistently obtain

10 support in more recent psychometric studies using exploratory and confirmatory factor

11 analyses in calibration and validation samples. The results suggested that it was necessary to

12 delete many items to improve the adjustment to the data, that a solution with more than two

13 factors was preferable and that some items were not sufficiently identified in their

14 corresponding factor. Adapted versions of the SPSRQ in different countries have reported

15 adequate convergent/discriminant validity measures with some regular personality measures,

16 but the empirical studies have also pointed out the need for the structural refinement of the

17 SPSRQ.

\subsection{Measurement instruments based on RST for children}

Although Gray's RST is of great interest in childhood, few studies have reported

21 evidence of the reliability/validity of measures based on this system in young children. Blair

22 (2003) developed a parent-report version for the assessment of RST in children based on the

23 BIS/BAS psychometric model, and in a sample of 42 children with a mean age of 4 he found

24 that the BIS-scale was positively related to teacher-reported social competence and the BAS- 
1 scale was not related to any of the study outcomes (physiological and cognitive self-

2 regulation, temperamental emotionality and social competence). Colder and O'Connor (2004) adapted items of the BIS/BAS and the SPSRQ to develop a new instrument for caregivers of 9- to 12-year-old children, the SPSRQ-C. Factorial analysis of this new tool in a sample of $n=63$ children aged 9-12 showed a good fit for a fourfactor solution: one dimension of the BIS (sensitivity to punishment) and three dimensions of the BAS (impulsivity/fun-seeking, drive and reward responsivity). The derived factors showed convergent validity with problem behaviors: high levels of impulsivity/fun-seeking (but not drive or reward responsivity) were related to high levels of externalizing scores, and high levels of sensitivity to punishment were related to high levels of internalizing scores. However, methodological issues limited this study's usefulness; sample size was low and no subsequent cross-validation data were available.

Most recently, Colder et al. (2011) reported the psychometric results of the parents' version of the SPSRQ-C in a sample of $N=387$ children aged 10-13, and concluded good reliability and validity for the seven-factor solution: fear/shyness, anxiety, conflict avoidance, sensory reward, drive, responsiveness to social approval and impulsivity/fun-seeking. The BIS and BAS scales showed cross-convergence and discriminant validity with other measures of the BIS-BAS dimensions, but while the BAS scales were related to physiological correlates, the BIS scales did not yield links to these outcomes). These results were also methodologically questionable, since two factors consisted of only two items (conflict avoidance and drive) and three factors obtained poor to moderate reliability (Cronbach's alpha of .45 for conflict avoidance, .52 for sensory reward and .65 for anxiety). Luman, van Meel, Oosterlaan and Geutrs (2012), in a large community sample of $N=1,234$ children aged 6 to 13 and based on the SPSRQ-C parents' reports, concluded that the best fit was provided by a four-factor solution: punishment sensitivity, reward responsivity 
1 and impulsivity with fun-seeking and drive. In this study, convergent-discriminant reliability

2 for the SPSRQ-C dimensions was obtained in the following clinical sub-samples of children

3 with disruptive problem behaviors: only-ADHD, $n=34$; ADHD with oppositional defiant

4 disorder -ODD-, $n=22$; and ADHD with autism spectrum disorder -ASD-, $n=22$; and a control

5 sample of typically developing children $(n=75)$. The results showed that: a) all ADHD groups

6 were characterized by high scores in reward responsivity and sensitivity to punishment; b)

7 ADHD-only and ADHD+ODD scored high on impulsivity fun-seeking and drive; and c)

8 ADHD+ASD scored high on punishment sensitivity.

9

\subsection{Justification of the study, objectives and hypotheses}

In short, early childhood is a malleable developmental period in which the detection of strengths and difficulties in sensitivity to punishment and reward has an enormous potential not only to re-shape these traits but also to detect endophenotypes or vulnerability factors of psychological disorders that could then be prevented. Dysfunctions in sensitivity to punishment and reward have been reported in several frequent disorders in childhood, such as ADHD, ODD, conduct disorder (CD) or anxiety disorders. However, methodological issues compromise the usefulness of many psychometric results, and more evidence of the structure and validity of RST measures is therefore needed at early ages.

This study aims to assess the psychometric properties of the SPSRQ-C in a large community sample of 6-year-old Spanish children. The specific objectives are: a) to validate the structure of the instrument through factor analysis; and b) to measure the association between the derived empirical SPSRQ-C factors and external dimensional and categorical measures of the children's temperament and psychological state. Based on the theoretical background, we hypothesize that: a) the internal structure of the Spanish version of the SPSRQ-C will be similar to the previous versions developed for childhood (the three- or four- 
1 factor solutions are the most final models); and b) the resulting derived factors will provide

2 evidence for Gray's RST: factors measuring sensitivity to punishment will be more strongly

3 related to internalizing psychological measures (especially with anxiety), while factors

4 measuring impulsivity and reward responsivity will obtain the strongest associations with

5 externalizing measures.

\section{Methods}

\subsection{Participants}

The sample derives from a longitudinal project on behavioral disorders in childhood designed in a two-phase sampling procedure (Ezpeleta, de la Osa \& Doménech, 2014). A total of 2,283 families, obtained from the census of all 3-year-old preschoolers in the Barcelona region $(N=13,578)$, were invited to participate, with 1,341 accepting the invitation. Sixty-three preschoolers were excluded due to language issues (children who were of foreign origin and they or their families did not speak Spanish fluently) or serious developmental problems (ASD, intellectual disability), and the remaining 1,278 were screened using the behavioral problems scale of the Strengths and Difficulties Questionnaire for parents of 3- to 4-year-olds (SDQ ${ }^{3-4}$; Goodman, 1997), plus four ODD symptoms (deliberately annoys people, blames others, is touchy, angry and resentful) from the Diagnostic and Statistical Manual of Mental Disorders (DSM-IV-TR; APA, 2000) not present in the questionnaire. With the aim of including children at high-risk of behavior problems, such as ODD, in the follow-up, screening was considered positive for raw scores $\geq 4$ on the $\mathrm{SDQ}^{3-4}$ conduct problems scale (which corresponds to percentile 90 in this scale), or a response option of 2 ('certainly true') for any of the 8 DSM-IV-TR ODD symptoms listed.

All preschoolers with a positive screening score were invited to participate $(N=522$ cases, $42.9 \%$ ), as well as a random $30 \%$ of the $N=756$ children with a negative screening 
1 score. The final sample at the end of the screening phase included 622 preschoolers (417 with

2 a positive screening score and 205 with a negative one). This study included data from

3 assessments at ages 3 and 6 . The sample of $N=622$ participants at age 3 included 311 boys

4 (50\%). Socioeconomic status (SES; Hollingshead, 1975) was distributed as follows: 205

5 (33.0\%) high-income families, 195 (31.4\%) medium-high, 88 (14.1\%) medium, 99 (15.9\%)

6 medium-low and $35(5.6 \%)$ low.

At age 6, complete data for the analyses carried out in this study were available for $N=483$ of the children who began the longitudinal study. No statistically significant

9 differences were found among participants and dropouts by the child's sex $\left(\chi^{2}=0.48, d f=1\right.$, $10 p=.49)$ or socioeconomic status $\left(\chi^{2}=8.39, d f=4, p=.08\right)$.

Regarding family composition, the mean number of members per family was 4.1

\subsection{Measures}

The Sensitivity to Punishment and Sensitivity to Reward Questionnaire for Children (SPSRQ-C; Colder \& O'Connor, 2004) was adapted to the Spanish population, combining back translation with a bilingual committee of experts (Hambleton, 1994). The original items were translated into Spanish by two bilingual clinical psychologists and the translated questionnaire was sent to and independent translator for back translation into English. The 
1 process comprised dual forward translations, both of which were performed by clinicians and

2 researchers in Spain with more than twenty years' expertise and experience in clinical and

3 research areas, as well as in the translation and validation of psychological measurement

4 instruments from English to Spanish populations. The two translators completed their

5 translation separately, after which two independent bilingual clinical psychologists (also with

6 great experience in clinical and research domains) reviewed the equivalence of the content.

7 Differences were discussed and a consensus reached. The resulting SPSRQ-C contains 33

8 items codified in a 5-point Likert scale (0: total disagree to 4: total agree). The data of this

9 study correspond to parents' reports on their children at age 6.

The Diagnostic Interview for Children and Adolescents for Parents of Preschool and

11 Young Children (DICA-PPYC; Reich \& Ezpeleta, 2009) is a semi-structured interview

12 adapted to the Spanish population (Ezpeleta, Osa, Granero, Doménech \& Reich, 2011), designed to assess DSM-5 (APA, 2013) mental disorders in children aged 3 to 7 as reported by parents. Average administration time is around 50 minutes. children's behavioral and emotional problems according to the perception of the parents, through 113 items recoded with a 3-point ordinal response scale (0: not true, 1: somewhat/sometimes true, 2: very true/often true). For this study, the Spanish adaptation was used, which demonstrated good psychometric properties (Sardinero, Pedreira \& Muñiz, 1997). The syndrome scales used as external clinical measures were: anxiety-depression, 21 attention problems, aggressive, internalizing, externalizing and total (Cronbach's alphas for 22 these scales in the sample are in Table 1). The Children's Behavior Questionnaire for 3-7 year olds (CBQ3-7; Rothbart, Ahadi, Hershey \& Fisher, 2001) assesses reactive and self-regulatory temperament through 94 items organized into 15 scales of the first order, which also makes it possible to obtain 3 overall 
1 dimensions of temperament: negative affectivity (discomfort, falling reactivity/soothability,

2 fear, anger/frustration, sadness), surgency (activity level, high intensity pleasure, impulsivity,

3 approach/positive anticipation, shyness) and effortful control (attentional focusing, inhibitory

4 control, low intensity pleasure, perceptual sensitivity, smiling/laughter). The Spanish

5 adaptation version was used for the study (de la Osa, Granero, Penelo, Doménech \& Ezpeleta,

6 2014), which achieved adequate psychometric properties. The information in this tool was

7 provided by the parents when their children were 5 years old. Scales selected as external

8 clinical measures for this study were activity level, anger-frustration, attentional focusing,

9 shyness and the second order scales (Cronbach's alphas in the sample are in Table 1).

\subsection{Procedure}

Approval was obtained from the Ethics Review Committee of the authors' institution

13

\subsection{Data analysis}

Confirmatory Factor Analysis (CFA) was conducted for the SPSRQ-C with MPlus7 for Windows. Solutions for one, two, three and four factors were tested using a robust estimator (Weighted Least Squares using a diagonal Weight Matrix (WLSMV) and the Delta 
1 parameterization. Goodness-of-fit was measured through Root Mean Square Error of

2 Approximation (RMSEA), Comparative Fit Index (CFI) and Tucker Lewis Index (TLI)

3 (adequate goodness-of-fit was considered good for RMSEA <.10, CFI $>.90$ and TLI $>.90$ )

4 (Kline, 2010). Internal consistency of the model-derived factors was measured through

5 Cronbach's alpha (higher consistency values are indicative of lower error variance).

The association between SPSRQ-C and external clinical measures was estimated by the Pearson correlation ( $r$, with effect size considered small for $|r|<.23$, medium for $.24<\mid r$ $\mid<.36$, and large for $|r| \geq .37$.

Logistic regressions analyzed the discriminative capacity of the SPSRQ-C to identify the presence of DSM-5 disorders according to the measurement of the diagnostic interview. Due to the strong association during childhood between children's sex and the presence of specific DSM-5 disorders, and since the aim of this analysis is to obtain the specific contribution of each SPSRQ-C factor on each DSM-5 disorder, logistic regressions were adjusted by the covariates children's sex and other comorbid disorders different to that included in the regression as the criterion. The goodness-of-fit for each model was measured through the Hosmer-Lemeshow test (good fit was considered for $p>.05$ ), the global predictive capacity through the Nagelkerke's pseudo- $\mathrm{R}^{2}$ coefficient, and the global accuracy of the classification with the area under the receiver operating characteristic (ROC) curve (AUC, considering adequate accuracy for AUC>.70) (Kleinbaum, Kupper, Nizam \& Rosenberg 2015).

Due to the multi-phase sampling design, all the analyses were weighted, assigning to each participant a weight equal to the inverse probability of selection in the second phase of sampling (after screening). 


\subsection{Factorial analysis}

Table S2 (supplementary) shows the standardized coefficients (loadings) for the oneto four-factor solutions in CFA modeling, the consistency for each factor $(\alpha)$, goodness-of-fit indexes and correlations between factors. The one-factor model was not considered since the fit was not adequate (RMSEA=.14, CFI=.57 and TLI=.54). This clearly improved for the twofactor model, although still remaining relatively poor: $\mathrm{RMSEA}=.08, \mathrm{CFI}=.85$ and TLI=.84. The best fit results were obtained for the three-factor (RMSEA=.08, CFI=.90 and TLI=.85) and the four-factor models (RMSEA=.08, CFI=.86 and TLI=.85). However, the four-factor model was not considered a good final candidate since two factors achieved consistencycoefficients lower than 0.80 and very high correlations were found between factor two with factors three $(r=.91)$ and four $(r=.87)$.

The final CFA selected in this work, therefore, was the three-factor model, which obtained a moderate to good fit: F1 sensitivity to punishment $(\alpha=.88)$, F2 impulsivity/funseeking and drive $(\alpha=.83)$, and F3 reward responsivity $(\alpha=.78)$. Table 1 includes CFA results for the three-factor model. Consistency was very good for factor F1 and F2, and good for F3 . F1 achieved medium correlations with F2 (r=.27) and F3 ( $r=.28)$, but F2 and F3 correlated highly $(r=.80)$.

Table S3 (supplementary) contains the distribution of the SPSRQ-C scores (means, standard deviations and percentiles for the factor scores) for the total sample, stratified by sex.

\subsection{Correlation between the SPSRQ-C factor scores and external clinical measures}

Table 2 contains the correlation between the SPSRQ-C factor scores and the external measures of psychopathology (CBCL) and temperament (CBQ). With regard to the level of psychological problems, F1 sensitivity to punishment yielded large positive associations with the CBCL anxiety, internalizing and total scales. F2 impulsivity/fun-seeking and drive and F3 
1 reward responsivity obtained sizable correlations with attention problems, aggressive

2 behavior, externalizing and total score, and medium correlations with anxiety-depression and 3 internalizing.

Regarding the children's temperament (measured at age 5), F1 sensitivity to punishment correlated positively with the CBQ anger/frustration scale, shyness and negative affectivity, and correlated negatively with surgency. F2 impulsivity/fun-seeking and drive correlated positively with activity level, anger-frustration and surgency, and negatively with attentional focusing and effortful control. F3 reward responsivity was positively related to activity level, anger/frustration and surgency, and negatively associated with attention focusing.

\subsection{Capacity of the SPSRQ-C factor scores to identify DSM-5 disorders}

Table 3 contains the logistic regression measuring the association between the SPSRQ-C factor scores and the presence of DSM-5 disorders. High scores in sensitivity to punishment (F1) were associated with high risk of the presence of ODD and any anxiety disorder. High levels of impulsivity/fun-seeking and drive (F2) increased the probability of disruptive disorders (ADHD, ODD). High scores in reward responsivity (F3) were not associated with any of the disorders considered in the study.

\section{Discussion}

This study provides evidence of the psychometric qualities of the SPSRQ-C (adapted from the original version by Colder and O'Connor, 2004) for 6-year-old children from the general population in Spain. CFA in a large community sample resulted in a final three-factor model with moderate to good goodness-of-fit. Internal consistency was very good for the 
1 the factor F3 reward responsitivity. The three factors showed theoretical/clinical

2 interpretability and they achieved convergent/discriminant validity with external measures of psychopathology (CBCL scales and DSM-5 diagnoses) and temperament (CBQ scales).

F1 sensitivity to punishment was particularly associated with internalizing problems:

large correlations were obtained with the CBCL anxiety-depression and internalizing scales, and significant discriminant capacity with the presence of any anxiety DSM-5 disorder. This result provides additional evidence of convergent/discriminant validity with BIS-related measures, particularly with anxiety. Behavioral inhibition in early childhood has been strongly related to subsequent development of anxiety disorders (Guyer et al., 2006; Kagan,

Schwartz, Wright, Shin, Kagan \& Rauch, 2003). The increased BIS functioning in children with high anxiety is manifested by the high levels of sensitivity to aversive stimuli of these children compared with their normal peers, and by their high sensitivity to punishment. Empirical studies outline that although most children lie to avoid punishment when they perceive that their behavior is inappropriate, children with high anxiety/depression sensitivity or high anxiety/depression levels would overestimate the magnitude of the misbehavior as well as magnitude of the punishment (Bijttebier, Beck, Claes \& Vandereycken, 2009). The factors F2 impulsivity/fun-seeking and drive and F3 reward responsivity achieved high associations with psychological externalizing problems. It has been argued that ADHD and other psychiatric conditions that usually co-occur with this diagnosis (disruptive disorders, and particularly ODD) show alterations in sensitivity to reward (Rommelse, Geurts, Franke, Buitelaar \& Hartman 2011). In a recent study conducted by Fosco, Hawk, Rosch and Bubnik (2015) with a sample of $N=58$ children (25 ADHD and 33 controls) aged 9-12, it was observed that the presence of the ADHD diagnosis was strongly related to higher scores in the response to reward trait (compared to the control group). Some experimental studies have also 
1 observed that ADHD or ADHD+ODD children are less sensitive to punishment than control

2 children (Luman, Sergeant, Knol \& Oosterlaan, 2010; van Meel, Oosterlaan, Heslenfeld \&

3 Sergeant, 2005) and that ODD children tend to maintain responses of sensitivity to reward

4 while ignoring the increasing rate of punishment (Matthys, van Goozen, Snoek \& Engeland,

5 2004; van Goozen et al., 2004). ADHD is characterized by serious difficulties in paying

6 attention and lack of motivation to finish tasks. Some studies suggest that these children

7 prefer small immediate rewards over large delayed rewards (Luman et al., 2012; Sonuga-

8 Barke, 2002).

Three main limitations of the study are: a) the inability to obtain multi-group CFA models to test the invariance of the factor structure by sex, ethnicity or socioeconomic-status (the sample size did not allow this modeling), which involve the need for future research to test if the structure for the SPSRQ-C obtained in this study is different depending on the precedence of samples and/or groups; b) the absence of cross-sectional measures for the CBQ and SPSRQ-C scales (registered at age 5 and 6), although it must be noted that developmental, biological and neuro-imaging studies highlight that the self-regulation construct is thought to develop most rapidly and crucially in very early life (the first four to five years of life; McClelland, Ponitz, Messersmith \& Tominey, 2010) and therefore a large significant clinical change is not expected between CBQ scores at age 5 and reactive and selfregulatory temperament one year later; and c) the absence of counterbalanced measures prevented a control for potential order effects (it must be also stated that to our knowledge no

21 previous study in the area was designed with this procedure, so our results are comparable to 22 other published research).

\section{Conclusion}


1 temperament traits in childhood) are a consequence of the individual differences in

2 neuropsychological system sensitivity to reward and punishment: BIS and BAS facilitate the

3 expression of aversive and appetitive motivation, and individual differences in both systems

4 underlie the personality dimensions of anxiety and impulsivity, respectively. The Spanish

5 version of the SPSRQ-C resulted in three factors with moderate to good psychometric

6 properties as a measure of the sensitivity to punishment and reward responsivity based on

7 RST. As a whole, our results are consistent with previous studies that also observed a high

8 proportion of common variance between behavioral manifestations of reward and punishment

9 systems and personality traits in children. The three empirically derived factors for the

SPSRQ-C in this study correlate with temperament traits assessed through the CBQ at age 5,

11 which provides additional evidence regarding the construct validity of RST itself in young

12 children.

Reliable and valid measurement of the RST systems is relevant in the areas of

educational and therapeutic interventions that use punishment and rewards as contingencies.

Thus, parent/teacher training programs may benefit especially.

\section{References}

Achenbach, T., \& Rescorla, L. (2001). Manual for the ASEBA school-age forms \& Profiles. Burlington, VT: University of Vermont, Research Center for Children, Youth \& Familie. [Inventario del Comportamiento de Niños/as de 6-18 años para Padres (CBCL/6-18)]

American Psychiatric Association. (2000). DSM-IV Diagnostic and statistical manual of mental disorders. Washington, DC: American Psychiatric Press.

American Psychiatric Association. (2013). Diagnostic and Statistical Manual of Mental Disorders, Fifth Edition (DSM-5). Washington, DC: American Psychiatric Association.

Ball, S., \& Zuckerman, M. (1990). Sensation seeking, Eysenck's personality dimensions and reinforcement sensitivity in concept formation. Personality and Individual Differences, 11, 343-345. 
Berkman, E., Lieberman, M., \& Gable, S. (2009). BIS, BAS, and response conflict: testing predictions of the revised reinforcement sensitivity theory. Personality and Individual Differences, 46, 586-591. doi: 10.1016/j.paid.2008.12.015.

Bijttebier, P., Beck, I., Claes, L., \& Vandereycken, W. (2009). Gray's reinforcement sensitivity theory as a framework for research on personality-psychopathology associations. Clinical Psychology Review, 29, 421-430. doi: 10.1016/j.cpr.2009.04.002.

Blair, C. (2003). Behavioral inhibition and behavioral activation in young children: Relations with self-regulation and adaptation to preschool in children attending headstart. Deviant Psychobiology, 42, 301-311.

Carver, C., \& White, T. (1994). Behavioral-inhibition, behavioral activation, and affective responses to impending reward and punishment-the BIS-BAS scales. Journal of Personality and Social Psychology, 67, 319-333.

Colder, C., \& O'Connor, R. (2004). Gray's reinforcement sensitivity model and child psychopathology: laboratory and questionnaire assessment of the BAS and BIS. Journal of Abnormal Child Psychology., 32, 435-451.

Colder, C., Trucco, E., López, H., Hawk, L., Read, J., Lengua, L., et al. (2011). Revised reinforcement sensitivity theory and laboratory assessment of BIS and BAS in children. Journal of Research in Personality, 45, 198-207. doi: 10.1016/j.jrp.2011.01.005.

Corr, P.J. (2004). Reinforcement sensitivity theory and personality. Neuroscience and Behavioral Reviews, 28, 317-332. doi: 10.1016/j.neubiorev.2004.01.005.

Corr, P.J. (2016). Reinforcement sensitivity theory of personality questionnaires: strutural survey with recommendations. Personality and Individual Differences, 86, 60-64. doi: 10.1016/j.paid.2015.09.045.

de la Osa, N., Granero, R., Penelo, E., Doménech, J.M., \& Ezpeleta, L. (2014). The Short and Very Short Forms of the Children's Behavior Questionnaire in a Community Sample of Preschoolers. Assessment, 21(4), 463-476. doi: 10.1177/1073191113508809.

Ezpeleta, L., de la Osa, N., \& Doménech, J. (2014). Prevalence of DSM-IV disorders, comorbidity and impairment in 3-year-old Spanish preschoolers. Social Psychiatry and Psychiatric Epidemiology, 49(1), 145-155. doi: 10.1007/s00127-013-0683-1.

Ezpeleta, L., de la Osa, N., Granero, R., Doménech, J., \& Reich, W. (2011). The Diagnostic Interview for Children and Adolescents for Parents of Preschool Children. Psychiatry Research, 190, 137-144. doi: 10.1016/j.psychres.2011.04.034.

Fosco, W., Hawk, L., Rosch, K., \& Bubnik, M. (2015). Evaluating cognitive and motivational accounts of greater reinforcement effects among children with attentiondeficit/hyperactivity disorder. Behavioral and Brain Functions, 11:20, doi: 10.1186/s12993-015-0065-9.

Goodman, R. (1997). The Strengths and Difficulties Questionnaire: A Research Note. Journal of Child Psychology and Psychiatry, 38, 581-586. doi: 10.1111/j.14697610.1997.tb01545.x.

Gray, J. (1970). The psychophysiological basis of introversion-extraversion. Behavior Research and Therapy, 8(3), 249-266. 
Gray, J. (1981). A critique to Eysenck's theory of personality. In H. Eysenck, A model for personality (pp. 246-276). New York: Springer.

Guyer, A., Nelson, E., Perez-Edgar, K., Hardin, M., Roberson-Nay, R., \& Monk, C.E. (2006). Striatal functional alteration in adolescents characterized by early childhood inhibition. Journal of Neuroscience, 26, 6399-6405. doi: 10.1523/JNEUROSCI.066606.2006.

Hambleton, R. (1994). Guidelines for adapting educational and psychological tests: A progress report. European Journal of Psychological Assessment, 10, 229-244.

Hollingshead, A. (1975). Four factor index of social status. Unpublished manuscript, Yale University, Department of Sociology, New Haven.

Kagan, J., Reznick, J., Snidman, N., Gibbons, J., \& Johnson, M. (1988). Childhood derivatives of inhibition and lack of inhibition to the unfamiliar. Child Development, $59,1580-1589$.

Keiser, H., \& Ross, S. (2011). Carver and Whites' BIS/FFFS/BAS scales and domains and facets of the Five Factor Model of personality. Personality and Individual Differences, 51, 39-44. doi: 10.1016/j.paid.2011.03.007.

Kleinbaum, D.G., Kupper, L.L., Nizam, A., \& Rosenberg, E. (2015). Applied regression analysis and other multivariate methods (5th ed.). Boston, MA: Cengage Learning.

Kline, R.B. (2010). Principles and practice of structural equation modeling (3rd ed.). New York, New York: Guilford Press.

Luman, M., Sergeant, J., Knol, D., \& Oosterlaan, J. (2010). Impaired decision making in oppositional defiant disorder related to altered psychophysiological responses to reinforcement. Biological Psychiatry, 68, 337-344. doi: http://dx.doi.org/10.1016/j.biopsych.2009.12.037.

Luman, M., van Meel, C., Oosterlaan, J., \& Geurts, H. (2012). Reward and punishment sensitivity in children with ADHD: validanting the Sensitivity to Punishment and Sensitivity to Reward Questionnaire for Children (SPSRQ-C). Journal of Abnormal Child Psychology, 40, 145-157. doi: 10.1007/s10802-011-9547-x.

Matthys, W., van Goozen, S., Snoek, H., \& van Engeland, H. (2004). Response perseveration and sensitivity to reward and punishment in boys with oppositional defiant disorder. European Child \& Adolescent Psychiatry, 13, 362-364. doi: http://dx.doi.org/10.1007/s00787-004-0395-x.

McClelland, M. M., Ponitz, C. C., Messersmith, E. E., \& Tominey, S. (2010). Self-regulation: Integration of cognition and emotion. In W. F. Overton \& R. M. Lerner (Eds.), The handbook of life-span development, Vol 1: Cognition, biology, and methods. (pp. 509553). Hoboken, NJ: John Wiley \& Sons Inc.

Morgan, B. (2006). Behavioral inhibition: a neurobiological perspective. Current Psychiatry Reports, 8, 270-278.

Reich, W., \& Ezpeleta, L. (2009). Entrevista Diagnóstica para Niños y Adolescentes Versión para Padres de Preescolares (3-7 años). Manuscrito no publicado. Barcelona: Unitat d'Epidemiologia i de Diagnòstic en Psicopatologia del Desenvolupament, Departament de Psicologia Clinica i de la Salut. UAB.

Rommelse, N., Geurts, H., Franke, B., Buitelaar, J., \& Hartman, C. (2011). A review on cognitive and brain endophenotypes that may be common in autism spectrum disorder 
and attention-deficit/hyperactivity disorder and facilitate the search for pleiotropic genes. Neuroscience and Biobehavioral Reviews, 35, 1363-1396. doi: 10.1016/j.neurobiorev.2011.02.015.

Rothbart, M. K., Ahadi, S. A., Hershey, K. L., \& Fisher, P. (2001). Investigations of temperament at 3-7 years: The Children's Behavior Questionnaire. Child Development, 72, 1394- 1408. doi:10.1111/1467-8624.00355.

Sardinero, E., Pedreira, J.L., \& Muñiz, J. (1997). El cuestionario CBCL de Achenbach: Adaptación española y aplicaciones clínico-epidemiológicas. Clínica y Salud, 8, 447480.

Schwartz, C. E., Wright, C. I., Shin, L. M., Kagan, J., \& Rauch, S. (2003). Inhibited and uninhibited infants "grown up": Adult amygdalar response to novelty. Science, 300, 1952-1953. doi: 10.1126/science.1083703.

Sonuga-Barke, E. (2002). Psychological heterogeneity in ADHD-a dual pathway model of behaviour and cognition. Behavioral Brain Research, 130, 29-36. doi: 10.1016/S0166-4328(01)00432-6.

Torrubia, R., Ávila, C., Molto, J., \& Caseras, X. (2001). The Sensitivity to Punishment and Sensitivity to Reward Questionnaire (SPSRQ) as a measure of Gray's anxiety and impulsivity dimensions. Personality and Individual Differences, 31, 837-862. doi: 10.1016/S191-8869(00)00183-5.

van Goozen, S., Cohen-Kettenis, P., Snoek, H., Matthys, W., Swaab-Barneveld, H., \& van Engeland, H. (2004). Executive functioning in children: a comparison of hospitalised ODD and ODD/ADHD children and normal controls. Journal of Child Psychology and Psychiatry, 45, 284-292. doi: 10.1111/j.1469-7610.2004.00220.x.

van Meel, C., Oosterlaan, J., Heslenfeld, D., \& Sergeant, J. (2005). Telling good from bad news: ADHD differentially affects processing of positive and negative feedback during guessing. Neuropsychologia, 43, 1946-1954. doi: 10.1016/j.neuropsychologia.2005.03.018.

Wilson, G.D., Barrett, P.T., \& Gray, J.A. (1989). Human reactions to reward and punishment: A questionnaire examination of Gray's personality theory. British Journal of Psychology, 80, 509-515. 
$1 \quad$ Table 1.

2 Standardized coefficients for the three-factor model (CFA).

\begin{tabular}{|c|c|}
\hline SPSRQ-C item & \\
\hline Original factor: sensitivity to punishment & $\mathrm{F} 1$ \\
\hline 02: Prefers not to ask for something & .322 \\
\hline 04: Frightened by new/unexpected situations & .706 \\
\hline 06: The child is shy & .711 \\
\hline 08: Avoids demonstrating skills for fear & .767 \\
\hline 10: Difficulties in speaking when in a group & .719 \\
\hline 12: Avoids going to places he/she doesn't know well & .706 \\
\hline 14: Concerned about the things that he/she said/did & .398 \\
\hline 16: Avoids talking to strangers & .713 \\
\hline 18: Avoids being with a group & .793 \\
\hline 20: Could do more things if he/she were not afraid & .781 \\
\hline 22: Afraid of more things than others are & .635 \\
\hline 24: Stops doing things to avoid rejection & .694 \\
\hline 26: Stops doing things for fear of embarrassment & .805 \\
\hline 28: Nervous, thinks something unpleasant happen & .529 \\
\hline 30: Hurt a lot by criticism or scolding & .463 \\
\hline Original factor: impulsivity/fun-seeking & $\mathrm{F} 2$ \\
\hline 13: Motivation for obtaining social recognition & .660 \\
\hline 17: Trouble resisting doing forbidden things & .642 \\
\hline 21: Difficulties in dropping a fun activity & .319 \\
\hline 23: Does things for immediate reward & .777 \\
\hline 25: Difficulties in sustaining attention (school) & .490 \\
\hline 27: The child gets involved in risky activities & .756 \\
\hline 33: Wants to experience new sensations & .647 \\
\hline \multicolumn{2}{|l|}{ Original factor: drive } \\
\hline 19: Likes to do everything he/she can to win & .751 \\
\hline 29: The child likes competitive activities & .716 \\
\hline 31: The child likes to be a socially powerful person & .693 \\
\hline 32: Likes to show off physical skills & .787 \\
\hline Original factor: reward responsivity & F3 \\
\hline 01: Obtaining rewards motivates the child & .427 \\
\hline 03: Often does things to be praised & .689 \\
\hline 05: Enjoys being the center of attention & .620 \\
\hline 07: Seeks to highlight when he/she is with a group & .633 \\
\hline 09: Feeling excited when he/she gets something & .407 \\
\hline 11: Does many things to get approval & .731 \\
\hline 15: Prefers activities with an immediate reward & .812 \\
\hline
\end{tabular}

3 F1: sensitivity to punishment; F2: impulsivity / fun seeking and drive; F3: reward responsitivity. 
$1 \quad$ Table 2.

2 Correlation between the SPSRQ-C and external clinical measures.

\begin{tabular}{|c|c|c|c|c|}
\hline & $\alpha$ & $\mathrm{F} 1$ & $\mathrm{~F} 2$ & F3 \\
\hline \multicolumn{5}{|l|}{ CBCL scales } \\
\hline Anxiety/depression & .73 & $.56^{\star \star}$ & $.30^{\star}$ & $.31^{*}$ \\
\hline Attention problems & .82 & .16 & $.49^{\star \star}$ & $.38^{\star \star}$ \\
\hline Aggressive behavior & .88 & .22 & $.43^{\star \star}$ & $.41^{\star \star}$ \\
\hline Internalizing & .82 & $.63^{\star \star}$ & $.26^{*}$ & $.26^{\star}$ \\
\hline Externalizing & .89 & .22 & $.47^{\star *}$ & $.42^{\star *}$ \\
\hline Total & .94 & $.41^{\star \star}$ & $.48^{\star \star}$ & $.45^{\star \star}$ \\
\hline \multicolumn{5}{|l|}{ CBQ scales (age 5) } \\
\hline Activity level & 0.76 & -.03 & $.44^{\star \star}$ & $.25^{\star}$ \\
\hline Anger/frustration & 0.76 & $.30^{\star}$ & $.28^{*}$ & $.26^{\star}$ \\
\hline Attentional focusing & 0.73 & -.06 & $-.32^{\star}$ & $-.24^{\star}$ \\
\hline Shyness & 0.83 & $.57^{\star \star}$ & -.02 & -.04 \\
\hline \multicolumn{5}{|l|}{$2^{\text {nd }}$ order scale } \\
\hline Surgency & 0.76 & $-.40^{* *}$ & $.41^{\star *}$ & $.24^{\star}$ \\
\hline Negative affectivity & 0.74 & $.37^{\star *}$ & .19 & .21 \\
\hline Effortful control & 0.75 & -.07 & $-.28^{\star}$ & -.14 \\
\hline
\end{tabular}

3 F1: sensitivity to punishment; F2: impulsivity / fun seeking and drive; F3: reward responsitivity.

$4 \quad{ }^{\star}$ Bold: medium ( $\left.|r| \geq .24\right)$ effect size. ${ }^{*}$ Bold: large effect size $(|r| \geq .37)$. $\alpha$ : Cronbach's alpha in sample.

5


1 Table 3.

2 Association between the SPSRQ-C and the presence of DSM-5 disorders: logistic regression adjusted by sex

3 and other comorbidity different to the criterion.

\begin{tabular}{|c|c|c|c|c|c|c|c|c|c|c|c|}
\hline \multirow{2}{*}{$\begin{array}{l}\text { Dependent variable } \\
\text { Any disruptive disorder }\end{array}$} & \multirow{2}{*}{$\begin{array}{l}\text { SPSRQ-C } \\
\text { F1 }\end{array}$} & \multirow{2}{*}{$\begin{array}{c}\text { B } \\
0.015\end{array}$} & \multirow{2}{*}{$\begin{array}{c}\text { SE } \\
0.015\end{array}$} & \multirow{2}{*}{$\begin{array}{l}\text { Wald } \\
1.03\end{array}$} & \multirow{2}{*}{$\begin{array}{c}p \\
310\end{array}$} & \multirow{2}{*}{$\begin{array}{l}\text { OR } \\
1.02\end{array}$} & \multicolumn{2}{|c|}{$95 \% \mathrm{Cl}(\mathrm{OR})$} & \multirow{2}{*}{$\begin{array}{l}\Delta \mathrm{R}^{2} \\
.178\end{array}$} & $\mathrm{H}-\mathrm{L}: \chi_{(\mathrm{df}=8)}^{2}(p)$ & \multirow{2}{*}{$\begin{array}{l}\text { AUC } \\
.756\end{array}$} \\
\hline & & & & & & & 0.99 & 1.05 & & $(.070)$ & \\
\hline & $\mathrm{F} 2$ & 0.112 & 0.026 & 18.07 & $<.001$ & 1.12 & 1.06 & 1.18 & & & \\
\hline & F3 & 0.045 & 0.042 & 1.15 & .284 & 1.05 & 0.96 & 1.14 & & & \\
\hline \multirow[t]{3}{*}{ ADHD } & $\mathrm{F} 1$ & -0.008 & 0.019 & 0.16 & .686 & 0.99 & 0.96 & 1.03 & .111 & $(.196)$ & .826 \\
\hline & $\mathrm{F} 2$ & 0.086 & 0.031 & 7.61 & .006 & 1.09 & 1.03 & 1.16 & & & \\
\hline & F3 & 0.069 & 0.051 & 1.82 & .177 & 1.07 & 0.97 & 1.18 & & & \\
\hline \multirow[t]{3}{*}{ ODD } & F1 & 0.047 & 0.019 & 6.25 & .012 & 1.05 & 1.01 & 1.09 & .101 & $(.531)$ & .829 \\
\hline & $\mathrm{F} 2$ & 0.089 & 0.034 & 7.04 & .008 & 1.09 & 1.02 & 1.17 & & & \\
\hline & F3 & 0.006 & 0.054 & 0.01 & .917 & 1.01 & 0.90 & 1.12 & & & \\
\hline \multirow[t]{3}{*}{ Any anxiety disorder } & $\mathrm{F} 1$ & 0.067 & 0.016 & 16.97 & $<.001$ & 1.07 & 1.04 & 1.10 & .090 & (.223) & .737 \\
\hline & F2 & -0.042 & 0.030 & 1.99 & .158 & 0.96 & 0.90 & 1.02 & & & \\
\hline & F3 & 0.044 & 0.046 & 0.93 & .335 & 1.05 & 0.96 & 1.14 & & & \\
\hline
\end{tabular}

4 F1: sensitivity to punishment; F2: impulsivity/fun-seeking and drive; F3: reward responsitivity.

5 ADHD: attention deficit hyperactivity disorder. ODD: oppositional defiant disorder.

$6 \Delta \mathrm{R}^{2}$ : Change in Nagelkerke's pseudo- $\mathrm{R}^{2}$.

7 H-L: Hosmer-Lemeshow test (goodness-of-fit statistic).

8 AUC: Area Under the ROC curve (accuracy measure).

9 Bold: significant association (.05 level).

10 
Psychometric properties of the SPSRQ-C

1 Table S1.

2 Sociodemographic and weighted prevalences of DSM-5 disorders $(N=483)$.

\begin{tabular}{|c|c|c|c|c|c|c|}
\hline \multicolumn{4}{|c|}{ Sociodemographics } & \multirow{2}{*}{$\begin{array}{l}\text { DSM-5 disorders } \\
\text { Any disruptive disorder }\end{array}$} & \multirow{2}{*}{$\begin{array}{l}\mathrm{N} \\
68\end{array}$} & \multirow{2}{*}{$\begin{array}{l}\text { (weighted \%) } \\
(12.7 \%)\end{array}$} \\
\hline Sex; $n(\%)$ & Female & 245 & $(50.7 \%)$ & & & \\
\hline \multirow[t]{5}{*}{ SES; $n(\%)$} & High & 164 & $(34.0 \%)$ & Attention-deficit-hyperactivity & 39 & $(7.7 \%)$ \\
\hline & Mean-high & 157 & $(32.5 \%)$ & Oppositional defiant disorder & 40 & $(6.9 \%)$ \\
\hline & Mean & 70 & $(14.5 \%)$ & Conduct disorder & 2 & $(0.3 \%)$ \\
\hline & Mean-low & 71 & $(14.7 \%)$ & Major depressive episode & 3 & $(0.4 \%)$ \\
\hline & Low & 21 & $(4.3 \%)$ & Any anxiety disorder & 44 & (8.6\%) \\
\hline \multirow[t]{3}{*}{ Ethnicity; $n$ (\%) } & Caucasian & 440 & $(91.1 \%)$ & Separation anxiety disorder & 7 & $(1.6 \%)$ \\
\hline & ic-American & 25 & $(5.2 \%)$ & Generalized anxiety & 6 & $(0.8 \%)$ \\
\hline & Other & 18 & $(3.7 \%)$ & Specific phobia & 26 & $(4.8 \%)$ \\
\hline \multicolumn{2}{|c|}{ Age (years-old); mean (SD) } & 6.61 & $(0.36)$ & Social phobia & 11 & $(2.2 \%)$ \\
\hline
\end{tabular}

3 SES: socioeconomic status. SD: standard deviation. 
1 Table S2.

2 Standardized coefficients for the one- to four-factor solutions in the CFA.

\begin{tabular}{|c|c|c|c|c|c|c|c|c|c|c|c|}
\hline \multirow{2}{*}{ SPSRQ-C item } & \multirow[t]{2}{*}{ CFA solution } & \multirow{2}{*}{$\begin{array}{c}1 \text {-fac. } \\
\text { F1 }\end{array}$} & \multicolumn{2}{|c|}{ 2-factors } & \multicolumn{3}{|c|}{ 3-factors } & \multicolumn{4}{|c|}{ 4-factors } \\
\hline & & & $\mathrm{F} 1$ & $\mathrm{~F} 2$ & $\mathrm{~F} 1$ & $\mathrm{~F} 2$ & F3 & $\mathrm{F} 1$ & $\mathrm{~F} 2$ & F3 & $\mathrm{F} 4$ \\
\hline \multicolumn{2}{|c|}{ Original factor: sensitivity to punishment } & & & & & & & & & & \\
\hline \multicolumn{2}{|c|}{ 02: Prefers not to ask for something } & .252 & .323 & & .322 & & & .321 & & & \\
\hline \multicolumn{2}{|c|}{ 04: Frightened by new/unexpected situations } & .608 & .706 & & .706 & & & .707 & & & \\
\hline \multicolumn{2}{|c|}{ 06: The child is shy } & .545 & .711 & & .711 & & & .709 & & & \\
\hline \multicolumn{2}{|c|}{ 08: Avoids demonstrating skills for fear } & .653 & .767 & & .767 & & & .766 & & & \\
\hline \multicolumn{2}{|c|}{ 10: Difficulties in speaking when in a group } & 601 & .719 & & .719 & & & .720 & & & \\
\hline \multicolumn{2}{|c|}{ 12: Avoids going to places he/she doesn't know well } & .592 & .707 & & .706 & & & .707 & & & \\
\hline \multirow{2}{*}{\multicolumn{2}{|c|}{$\begin{array}{l}\text { 14: Concerned about the things that he/she said/did } \\
\text { 16: Avoids talking to strangers }\end{array}$}} & .340 & .398 & & .398 & & & .399 & & & \\
\hline & & .557 & .713 & & .713 & & & .712 & & & \\
\hline \multicolumn{2}{|c|}{ 18: Avoids being with a group } & .675 & .793 & & .793 & & & .792 & & & \\
\hline \multicolumn{2}{|c|}{ 20: Could do more things if he/she were not afraid } & .692 & .781 & & .781 & & & .781 & & & \\
\hline \multicolumn{2}{|c|}{ 22: Afraid of more things than others are } & .524 & 635 & & .635 & & & .638 & & & \\
\hline \multicolumn{2}{|c|}{ 24: Stops doing things to avoid rejection } & .622 & .694 & & .694 & & & .695 & & & \\
\hline \multicolumn{2}{|c|}{ 26: Stops doing things for fear of embarrassment } & .707 & .805 & & .805 & & & .805 & & & \\
\hline \multicolumn{2}{|c|}{ 28: Nervous, thinks something unpleasant happen } & .497 & .528 & & .529 & & & .530 & & & \\
\hline \multicolumn{2}{|c|}{ 30: Hurt a lot by criticism or scolding } & .415 & .463 & & .463 & & & .460 & & & \\
\hline \multicolumn{12}{|c|}{ Original factor: impulsivity/fun-seeking } \\
\hline 13: Motivation for o & ining social recognition & .552 & & .646 & & .660 & & & .648 & & \\
\hline 17: Trouble resistin & loing forbidden things & .535 & & 627 & & 642 & & & .628 & & \\
\hline 21: Difficulties in $d r$ & bing a fun activity & .295 & & .312 & & .319 & & & .318 & & \\
\hline 23: Does things for & mediate reward & 676 & & .755 & & .777 & & & .762 & & \\
\hline 25: Difficulties in su & ining attention (school) & .419 & & .478 & & .490 & & & .482 & & \\
\hline 27: The child gets it & Ived in risky activities & .642 & & .739 & & .756 & & & .739 & & \\
\hline 33: Wants to experi & ce new sensations & .523 & & .631 & & .647 & & & .629 & & \\
\hline Original factor: driv & & & & & & & & & & & \\
\hline 19: Likes to do eve & ing he/she can to win & .600 & & .734 & & .751 & & & & .803 & \\
\hline 29: The child likes & npetitive activities & .553 & & .698 & & .716 & & & & .765 & \\
\hline 31: The child likes $t$ & e a socially powerful person & .585 & & .677 & & .693 & & & & .733 & \\
\hline 32: Likes to show o & hysical skills & .672 & & .770 & & .787 & & & & .860 & \\
\hline Original factor: rew & responsivity & & & & & & & & & & \\
\hline 01: Obtaining rewar & motivates the child & .304 & & .389 & & & .427 & & & & .428 \\
\hline 03: Often does thin & to be praised & .542 & & .629 & & & .689 & & & & .689 \\
\hline 05: Enjoys being th & enter of attention & .414 & & .571 & & & .620 & & & & .621 \\
\hline 07: Seeks to highlig & when he/she is with a group & .424 & & .586 & & & .633 & & & & .633 \\
\hline 09: Feeling excited & len he/she gets something & .280 & & .373 & & & .407 & & & & .405 \\
\hline 11: Does many thin & to get approval & .608 & & 671 & & & .731 & & & & .731 \\
\hline 15: Prefers activitie & ith an immediate reward & .656 & & .732 & & & .812 & & & & .810 \\
\hline & sistency: Cronbach's alpha $(\alpha)$ & .887 & .884 & .878 & .884 & .832 & .770 & .884 & .738 & .761 & .770 \\
\hline Fitting: RMSEA & Correlations: F1-F2 F2-F3 & .135 & .080 & .29 & .079 & .27 & .80 & .078 & .34 & .91 & \\
\hline $\mathrm{CFI}$ & $F 1-F 3 \quad F 2-F 4$ & .569 & .850 & & .900 & .28 & & .859 & .16 & .87 & \\
\hline TLI & $F 1-F 4 \quad F 3-F 4$ & .541 & .840 & & .854 & & & .848 & .28 & .68 & \\
\hline
\end{tabular}

3 Italics: correlation between factor scores. 
Psychometric properties of the SPSRQ-C

1 Table S3.

2 Distribution of the SPSRQ-C scores.

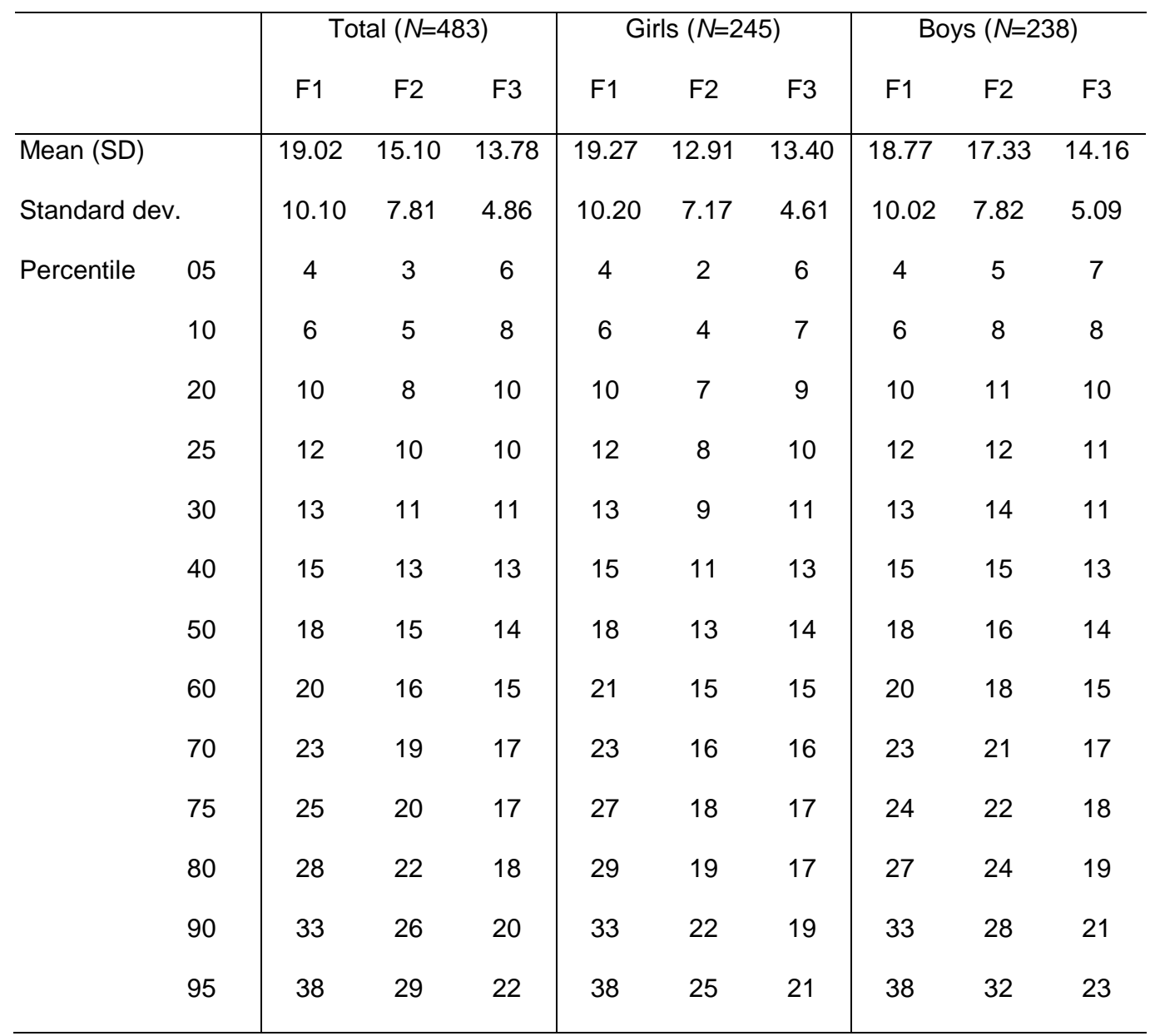

F1: sensitivity to punishment; F2: impulsivity/fun-seeking and drive; F3: reward responsitivity. 